\title{
Energy Efficient QoS guaranteed Cross Layer Solution (EEQCL) for Mesh Backbone IOT Networks
}

\author{
Gauri Sameer Rapate, Naveen N C
}

\begin{abstract}
IOT enables devices to be managed and monitored anywhere from internet. With IOT gaining popularity, billions of devices are connected to internet and they are being used for many applications like home automation, public safety, smart city, traffic monitoring etc. IOT interconnectivity to internet based on mesh network is the most used topology due to effectiveness in scalability and reliability. The mesh network must be optimized in terms of QOS, energy efficiency for the case of nature of traffic from IOT devices. Towards this end, this work proposes an energy efficient QOS guaranteed cross layer solution for mesh backbone based IOT network. The proposed cross layer solution applies changes at Application, session, network layer to achiness a better QOS and energy efficiency than existing solutions discussed in literature.
\end{abstract}

\section{INTRODUCTION}

Internet of Things (IoT) allows devices to be connected to internet for realization for various applications such as smart industries applications, smart cities, smart agriculture, smart health, etc. Growth in semiconductor industry has proliferated small devices with powerful processing ability and network capabilities getting connected to IOT network in larger scale. The existing computer based networks for integrating IOT devices to internet has many challenges. First challenge is that these computer based networks are not designed for low power sensor devices as the result battery energy is exhausted at faster rate and device becomes dead soon. Second challenge is that networks are not adaptive to the faulty nature of the sensor devices. Due to battery constraints use of cellular or satellite infrastructure for connecting IOT devices to internet is not efficient. Wireless Mesh Network (WMN) is the way forward for connecting IOT devices to internet. A Communication network I a mesh topology which is formed of radio nodes is called as Wireless Mesh Network (WMN). One of most important advantage in using of WMN for IOT networks is its versatility. New routers can be added effortlessly in WMN thereby capacity and range of the network can be expanded without introducing more cables and connections. Apart from scaling and pricing advantages, WMN can also provide fault tolerance in case of disasters. Under failures, the network can reconfigure itself and provide continuous service. Use of WMN for IOT has many disadvantages in terms of unreliable

Revised Manuscript Received on October 25, 2019

Gauri Sameer Rapate, Department of Information Science and Engineering, Vemana Institute of Technology, VTU, India

Dr. Naveen N C, Department of Computer Science and Engineering, JSSATE, VTU, India

Email:gauri.rapate@gmail.com,ncnaveen@gmail.com
QOS. Congestions and failure in network disrupts the QOS. The energy factor of IOT devices and WMN must be optimized for better longevity of the network. Towards this end many routing protocols are available in literature. These challenges in WMN based IOT be met with single layer solutions alone and thus it motivates to design a cross layer based solution to model the QOS and energy consumption in terms of multiple parameters at different layer of OSI and optimize those parameters. An energy efficient QOS guaranteed cross layer solution is proposed in this work. With control at application, session and network layers, application specific QOS with optimal energy consumption is managed effectively for WMN based IOT networks. Following are the components of the proposed cross layer solution.

1. Tree based topology optimized for ensuring better QOS and reduced energy consumption.

2. Application specific delay tolerant packet scheduling and network overhead reduction.

3. Application and Network adaptive packet rate adjustment at session layer.

\section{RELATED WORK}

In [1] author proposed a model of cross layer transmission designed for IoT based on wireless adhoc networks. This model applied techniques like decentralized coded caching and content division multiplexing in the physical layer and media access control layer respectively. The solution works best for a specific traffic model of alternating high and low traffic periods. The work reduced network congestion and was able to reduce the delay, but energy consumption is not considered. Routing by Energy and Link quality (REL) for IOT is proposed in [2]. The work proposed an end to end link quality estimator mechanism and used it with residual energy and hop count for the purpose of route selection. Furthermore the work proposed an event driven mechanism for load balancing and avoiding energy depletion of nodes. The link quality estimator mechanism is heavily dependent on network topology. Similar to [2] authors in [3] proposed a Distance, Energy and Link quality based Routing protocol (DELR) for IOT to improve the packet success ratio. Link quality is calculated in terms of RSSI (Radio Signal Strength Indicator) and the AODV routing protocol messages is added with the estimated link quality indicator value. Based on threshold poor quality links are filtered in the routing. 
Authors in [4] proposed an energy-efficient probabilistic routing (EEPR) algorithm. The work applies energy efficient probabilistic control on RREQ using the residual energy of each node and ETX metric of AODV protocol. The solution is able to improve the network lifetime of AODV protocol. The solution considers only reduction in routing overhead but does not consider energy efficiency in data forwarding phase. Authors in [5] proposed a new energy-efficient routing protocol using message success rate. The routing path is selected in cluster based topology using message success rate as the optimization criteria. For energy efficiency, a novel cluster head selection and cluster maintenance protocol is proposed. Routing path with better energy consumption is selected in [6]. Among all the routing paths, path with maximum available power (MAP) is selected in this work. Through this way energy fairness for network is ensured but delay and congestion is higher in this solution. Authors in [7]

Have proposed a technique that provides reliable energy efficient packet delivery. Here, set of nodes are made a Reference Nodes (RNs) by providing location-awareness capability to them. Now, the network area is segmented into few regions with the help of RN. Using Self Rejoining algorithm, Region Code ( $\mathrm{RC}$ ) is computed. RC with region-based route discovery discovers route between source and destination. Disadvantages of their proposed work is that, firstly it is completely simulation based, secondly the quality of nodes regarding battery life is not considered. The RPL protocol is the IETF proposed standard protocol for IPv6-based multi-hop wireless sensor networks (WSN). The RPL requires that the communication paths pass through a central router that can provide subroutine routes, leaving no distinction between the applications run by nodes. In [8] author proposed an application driven extension to RPL with a goal to increase the life time of WSN. It does so by limiting the routing and forwarding functions of the network. A novel RPL implementation is proposed in [9]. It minimizes the carbon footprint emissions without compromising on the delay and energy efficiency. The work selects the parent node in the routing path based on optimization of energy, carbon emission with constraints on delay. The solution models the delay in terms of link quality expressed in terms of energy cost for retransmissions but does the consider the number of routing paths sharing that link and congestion level. Due to this, delay constraints violation is higher in this approach. Authors in [10] proposed a novel energy efficient region-based routing protocol, called ER-RPL. It attains reliable and energy efficient data delivery. The salient feature in this solution is that route discovery involves only a subset of total nodes in the network. Author in [11] proposed a cross layer scheme for routing mechanism named CLB (Cost Link Based) that efficiently routes the traffic inside the WSN which eventually improves network lifetime. CLB uses multiple paths between the sensor node and the sink node, thereby balancing the traffic inside the wireless sensor network. Depending on network topology, the link distance can be calculated and the link cost highly depends on cross layer design that neglects paths with nodes that have limited energy over a specified range. A novel cross layer QOS aware routing protocol extending OLSR is proposed in [12]. Here, author gave proposal for two routing mechanisms by constructing multi-layer virtual logical mapping over physical topology. With the help of topology and bandwidth information, optimized routing path is identified. But the paths are not energy efficient. Authors in [13] proposed a new routing protocol for IOT considering context-aware computing into the basis routing protocol. Using fuzzy match theory of artificial intelligence and Markov probability mode in making forwarding decisions authors transformed the traditional routing thought into a positive predictable routing protocol. But the approach is not scalable and it is not energy efficient. A novel deployment scheme is proposed in [14] for addressing the energy efficiency issues in IOT. A hierarchical network design for energy efficient IOT and a minimum energy consumption transmission algorithm is proposed in this work. Heterogeneity is not taken into consideration for design of the network topology in this work. An energy-efficiency hierarchical clustering index tree and time correlated region query is proposed for energy efficiency in WSN based IOT network in [15]. The approach is able to achieve energy efficiency by reducing the redundant transmissions but does not consider the delay caused at aggregation centers. Authors in [16] proposed two strategy of low reliability requirement with shorter transmission distance to reduce the energy consumption of nodes in the hotspot area. The deployment model is optimized to ensure reduced energy consumption and in case of failure of nodes, the packet delivery ratio is very poor in this approach. Authors in [17] have proposed a delay-aware network structure for WSNs with in-network data fusion. For each cluster to communicate with the fusion center author has proposed a structure that arranges sensor nodes of different sizes in an interleaved manner. An optimization process is also proposed by authors to optimize intra-cluster communication distance. The authors have used a data aggregation model which is based on time-slot. Here a single time-slot is required to transmit data packet. However, number of time slots increases when there is increase in size of the fused data packet by a certain factor. TDMA was used at the MAC layer with the assumption that nodes are synchronized at time-slot and time cycle levels. For smooth communication, parent nodes will use separate spreading sequence and a dedicated time slot will be assigned to child nodes by parent node. The authors have proved through simulations that in their proposed network structure delay is reduced by around 2 time slots in data aggregation process and if the data is partially fused the total energy consumption is low. As packet arrival rate plays an important role in data fusion of cluster based WSN's it should be considered which is not done here. Authors in [18] solved the routing and bandwidth allocation problem as a linear optimization problem. In proposed network utility metric throughput and residual energy are taken into account. This approach does not consider application nature but to optimize the network usability it uses routing tree construction and bandwidth flow assignment. An efficient cross layer mechanism for multimedia data gathering in proposed in [19]. In this adaptive scheme the transmission Radius is changed dynamically and data generation Rate Adjustment (RRA) is based on a cross-layer design.

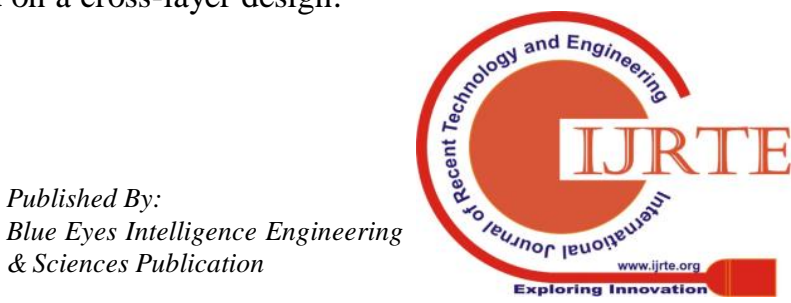


RRA reduces the transmission delay in WSNs while using the minimum data generation rate where an optimal transmission radius can be derived. Data generation rate can be adjusted by RRA to increase data gathering through transmission radius. Data gathering performance is increased in WSN's with RRA scheme by changing transmission radius of sensor nodes and the data generation rate of source nodes. The only disadvantage of this approach is that nature of application and their preferences are not taken into consideration. An approach for clustered WSN is proposed in [20] based on cross layer QoS provision. To support heterogeneous flow it classifies WMSN traffic into six traffic classes. To provide QoS in WMSNs this approach implements a service differentiation module and for every traffic class a unique data forwarding mechanism is proposed. To achieve the main goal that is to maximize network usage and avoid starvation of low priority flows, the priority of the traffic class determines the forwarding behavior, with the service differentiation module allocating bandwidth to each traffic class. When the data rate is estimated the characteristics of different traffic classes as well as their total bandwidth usage is considered by the congestion control mechanisms.

\section{PROPOSED EEQCL SOLUTION}

The proposed energy efficient QoS guaranteed cross layer solution involves topology based energy efficient QOS guaranteed routing using feedback from link layer queues, Data rate control based on RTT measurement at Application layer, Differentiated service flow based flow processing at Session layer and power control with multi wireless interface at physical layer.

\section{A. Topology based energy efficient QoS guaranteed routing}

Clustering based topology is adopted in the proposed solution. Every mesh node works on two wireless interfaces. One interface is used for communication between mesh nodes and other interface for communication with IOT devices. IOT devices and mesh nodes are heterogeneous with mesh nodes of higher storage capacity. The network is clustered using any density based clustering mechanisms with many IOT devices and mesh nodes in the cluster. The mesh node with maximum number of mesh nodes as its neighbors within the cluster is selected as Cluster head. There are two modes of routing intra cluster and inter cluster. The proposed routing mixes both of reactive and proactive routing to achieve energy efficiency and QOS guarantee. Reactive mode is used for intra cluster and proactive mode is applied for inter cluster routing. Reactive mode at intra cluster, selects the paths based on path score factor (PSF). Path score factor is calculated based on the residual energy of nodes in the path and estimated end to end delay. Path with a higher path score factor is selected for routing. PSF is calculated as

$P S F=\alpha \times D F+(1-\alpha) \times R F$

Where DF is the delay factor and RF is the residual energy factor. DF is calculated as

$D F=\sum_{i=1}^{n}\left\{\left(n p_{i}+1\right) \cdot\left(\frac{1}{1-p_{i}}\right) \cdot p t_{i}\right\}$

Where $n p_{\mathrm{i}}$ is the number of packets in queue at link $\mathrm{i}, P_{\mathrm{i}}$ is the transmission failure probability at link $\mathrm{i}$ and $p t_{\mathrm{i}}$ is the packet transmission time over link $i$.

The packet transmission time over link can be modeled in terms of influence on maximum throughput as

$p t_{\mathrm{i}}=\frac{L}{\left(1-R_{\mathrm{i}}\right) B_{\mathrm{i}}}$

Where $B_{i}$ is the channel bandwidth for link $\mathrm{i}$ under the influence of interference and $\mathrm{L}$ is the packet size. The net bandwidth usage is expressed as $\left(1-R_{i}\right)$.

$\mathrm{RF}$ is calculated as

$R F=\frac{\min \left(r \theta_{i}, r e_{j}\right)}{I E}$

Where $r e_{i}$ is the residual energy at link i.

AODV routing protocol is modified to incorporate $D F$. Every time RREP is forwarded, each node calculates it parts of DF and adds the new field DF added into RREP message. The source node which originate the RREQ message, selects the best RREP route with highest values of DF and use it for forwarding the packets within cluster. The RREQ is forwarded only to nodes within the same cluster. This can be controlled by checking the destination field in the RREQ message which is nothing but the cluster id. Due to control of area of forwarding of RREQ, the number of messages in the network is reduced and this further reduces the energy.

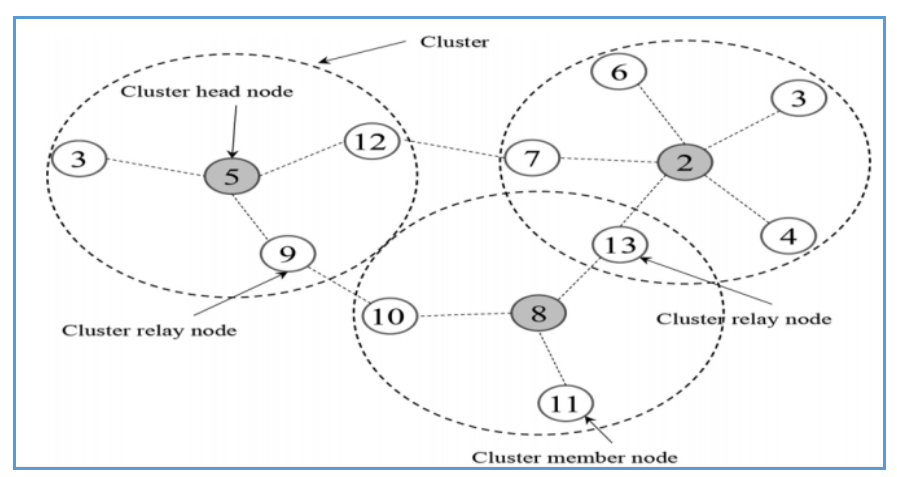

Figure 1 Cluster topology

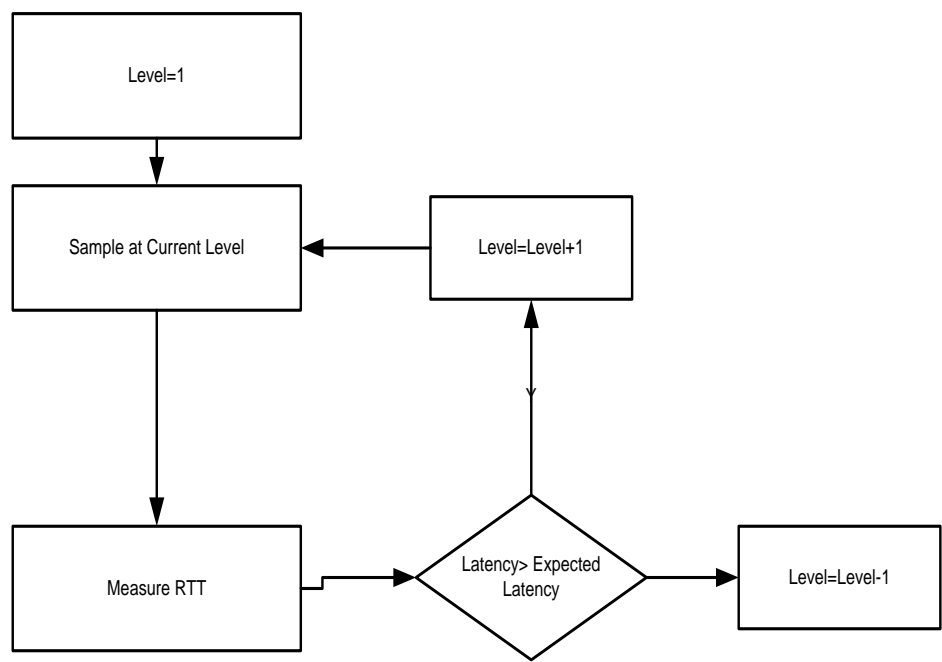

Fig: Adaptive Sampling

consumption due to forwarding of routing messages.

Cluster heads use proactive routing mode to route the packets

to Gateway. Using of a separate channel for routing the message 
across cluster relay to gateway helps to achieve the QOS guarantee. The proactive mode uses OLSR based link cost update. Each cluster head selects mesh nodes at boarder as relay nodes and these relay node send 2 hop link status cost to neighboring cluster. The link cost is updated in terms of average delay from the relay node to sink. The average delay is estimated using Mean Exponentially Weighted Moving Average Algorithm. $\psi=\alpha * \psi+(1-\alpha) * D$

Where $\alpha$ is in range of 0 to 1 and $\mathrm{D}$ is the last observed delay for packet transmission from relay node to sink.

\section{B. Data rate control at Application Layer}

Data rate control for text data is done by adjusting the sampling level of data collection by the IOT devices. IOT devices have multiple sampling levels which are arranged from highest to lowest. At highest level of sampling more number of packets is generated from IOT devices. Initially the devices start with highest number of samples and adjust the level dynamically based on the round trip time feedback. The RTT is modeled as probability mass function of delay distribution as The RTT is modeled as probability mass function of delay distribution as

$$
R T T=\left\{\begin{array}{c}
\sum_{i=0}^{\infty} f_{i}(a) \cdot f_{i}(b), x=0 \\
\sum_{i=0}^{\infty} f_{i}(a) \cdot f_{2 x+i}(b)+\sum_{i=0}^{\infty} f_{i}(b) \cdot f_{2 x+i}(a), x>0
\end{array}\right.
$$

Where $a, b$ are forward and backward directions from transmitter to receiver and $f(z)$ is the probability mass function of delay of direction $\mathrm{z}$.

Data rate control for image data is done by up scaling or down scaling the image using Laplacian Gaussian transformation. Gaussian and Laplacian pyramid of the original image is constructed. The reduced resolution levels of the Gaussian pyramid are themselves obtained through a highly efficient iterative algorithm. The bottom, or zero level of the pyramid, $G_{0}$, is the original image. This is low pass-filtered and sub sampled by a factor of two to obtain the next pyramid level $G_{1}$. The levels of pyramid is obtained

$$
G_{\mathrm{i}}(i, j)=\sum_{m} \sum_{n} w(m, n) G_{\mathrm{i}-1}\left(2 i+m_{v} 2 j+n\right)
$$

The weighing function $w\left(m_{v} n\right)$ is called as generating kernel. The difference between the successive levels of the Gaussian pyramid is represented as Laplacian pyramid. $L_{\mathrm{i}}=G_{\mathrm{i}}-G_{\mathrm{i}-1}$

$\prod_{T=Q}^{Q+4}(S Q-C Q)<D e V$

For each of the Gaussian pyramid level, the memory size of image is calculated and divided by the integrated compression ratio (IC) to obtain the network size. The value of integrated compression ratio is obtained by compressing some sample images with neural compression and predictive coding and averaging them. The network size of the image divided by IC gives the latency for transmission of image from transmitter to receiver. The level of Gaussian pyramid for which the latency meets the expected latency value for transmission is set as level of operation.

\section{Differentiated Service flow based processing}

Each packet generated by the application is categorized to one of two priority flows real time and non-real time and flow control is realized at the session layer. At each of cluster relay node, flow control of these two service flow is done. Each relay node estimates the traffic demand for relaying the packets from its neighbor clusters and allocates the timeslots proportional to the traffic demand and priority of the packets from the clusters. This process is repeated at beginning of each time frame. The future traffic demand (FD) for a neighbor node is estimated as

$F D=\min \left(M A_{\mathrm{i}}+D_{\mathrm{i}}, T \times \Delta\right)$

Where, $M A$ is the exponential moving average of incoming traffic to node, $\mathrm{D}$ is the traffic amount in the buffer and $\mathrm{T}$ is the current physical transmission rate. MA is calculated as $M A_{\mathrm{i}}=\left\{\begin{array}{c}\alpha \cdot T+(1-\alpha) M A_{\mathrm{i}-1} \text { if } T \neq 0 \\ (1-\alpha) M A_{\mathrm{i}-1,1} \text { otherwise }\end{array}\right.$

The traffic demand is calculated for each neighbor node sending the packet and slots ns for node $\mathrm{x}$ is allocated from total of $\mathrm{N}$ as

$n s_{x}=\frac{F D_{x}}{\sum_{x=1}^{n} F D_{x}} * \frac{P_{x}}{\sum_{x=1}^{n} P_{x}}$

Where $\mathrm{P}$ is the priority of packet from node $\mathrm{x}$. Based on packet priority information from the application layer and estimated traffic demand, the slots are allocated dynamically.

\section{Power control at Physical layer}

At each of the cluster relay node, transmission power control is done to reduce the energy consumption and increase the life time of the network. Based on the measured values of RSSI (Radio Signal Strength Indicator) and LQI (Link Quality Indicator) transmission power control is decided. When there is no transmission signal strength is sampled periodically to determine the base noise $\left(\mathrm{N}_{\mathrm{B}}\right)$. Communication between nodes $\mathrm{A}$ to node $\mathrm{B}$ will start by transmitting first packet at the current transmission power $\left(\mathrm{P}_{\mathrm{TX}}\right)$. The packet should have $\mathrm{P}_{\mathrm{TX}}$ on its headers. Once the packet is received by node $\mathrm{B}$, it calculates ideal transmission power $\left(\mathrm{P}_{\text {TXideal }}\right)$ from $\mathrm{A}$ to $\mathrm{B}$ using below equation $P_{T X}=\max \left(\frac{R X_{\text {thrsshold }}}{G_{A \rightarrow B}}, \frac{S_{\text {thingsholdx }} N_{B}}{G_{A \rightarrow B}}\right)$ and also determines signal strength $\left(\mathrm{P}_{\mathrm{RX}}\right.$, or reception power).

Each cluster relay node calculates the $P_{T X}$ for every other cluster relay node which it communicates and selects the maximum value as the transmission power for its operation. Relaying is reduced by limiting the transmission power, energy consumption for.

\section{RESULT}

The proposed EEQCL solution was simulated in NS2. Simulation was conducted with following parameters 


\begin{tabular}{|l|l|}
\hline Parameters & Values \\
\hline Number of Nodes & 500 \\
\hline $\begin{array}{l}\text { Communication } \\
\text { range }\end{array}$ & $100 \mathrm{~m}$ \\
\hline Area of simulation & $1200 \mathrm{~m} * 1200 \mathrm{~m}$ \\
\hline $\begin{array}{l}\text { Priority } \\
\text { distribution }\end{array}$ & $\begin{array}{l}20 \% \text { of packets generated by each node is } \\
\text { designated real time }\end{array}$ \\
\hline $\begin{array}{l}\text { Node Deployment } \\
\text { Topology }\end{array}$ & Random \\
\hline Simulation time & 10 minutes \\
\hline $\begin{array}{l}\text { Interface Queue } \\
\text { Length }\end{array}$ & 50 \\
\hline MAC & 802.11 \\
\hline $\begin{array}{l}\text { Number of Base } \\
\text { station }\end{array}$ & 1 \\
\hline $\begin{array}{l}\text { Location of Base } \\
\text { station }\end{array}$ & Upper right \\
\hline $\begin{array}{l}\text { Initial energy of } \\
\text { nodes }\end{array}$ & 100 joules \\
\hline Data traffic & CBR with each packet of size 512 bytes \\
\hline
\end{tabular}

The proposed solution is compared with two other cross layer architectures proposed in [1] and [19] and detailed in survey. We measured following parameters

\section{Throughput}

2. Delay

3. Packet Delivery Ratio

4. Network Lifetime

5. Network life time due to transmission control

6. Network life time due to data rate control

The throughput at gateway (kbps) is measured for different number of nodes and result is given below

\begin{tabular}{|l|l|l|l|}
\hline Nodes & {$[1]$} & {$[19]$} & Proposed \\
\hline 100 & 182 & 185 & 197 \\
\hline 200 & 191 & 193 & 206 \\
\hline 300 & 193 & 201 & 211 \\
\hline 400 & 197 & 209 & 220 \\
\hline 500 & 198 & 211 & 227 \\
\hline
\end{tabular}

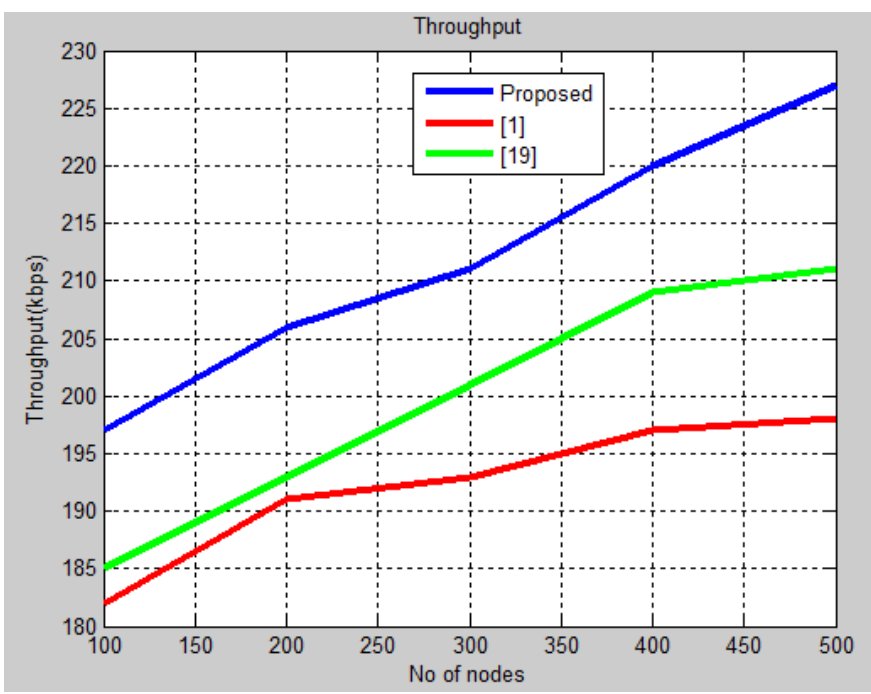

The proposed solution has higher throughput than [1] and [19]. The higher throughput is attributed to shorter hop for the travel of packet from node to sink.
The average delay (sec) is measured for different number of nodes and the result is given below

\begin{tabular}{|l|l|l|l|}
\hline Nodes & {$[1]$} & {$[19]$} & Proposed \\
\hline 100 & 0.12265 & 0.10023 & 0.0812 \\
\hline 200 & 0.12983 & 0.10114 & 0.0991 \\
\hline 300 & 0.13898 & 0.11291 & 0.10453 \\
\hline 400 & 0.18261 & 0.13062 & 0.11332 \\
\hline 500 & 0.20122 & 0.17366 & 0.14222 \\
\hline
\end{tabular}

The proposed solution has lower delay than [1] and [19]. The reduction in delay is faster propagation within and across clusters.

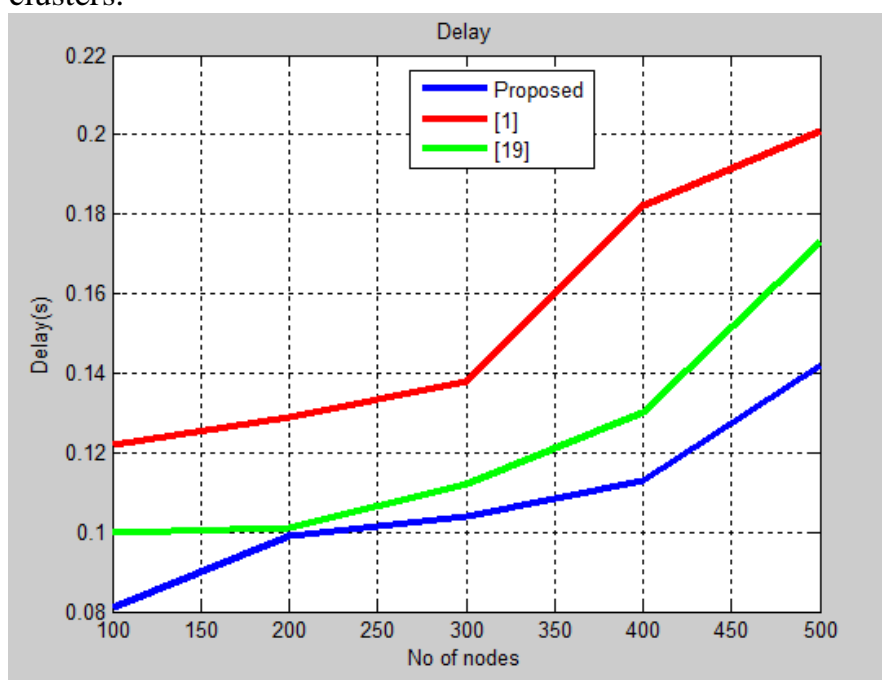

The packet delivery ratio is measured for different number of nodes and result is given below

\begin{tabular}{|l|l|l|l|}
\hline Nodes & {$[1]$} & {$[19]$} & Proposed \\
\hline 100 & 0.775 & 0.813 & 0.932 \\
\hline 200 & 0.791 & 0.803 & 0.931 \\
\hline 300 & 0.785 & 0.805 & 0.942 \\
\hline 400 & 0.764 & 0.808 & 0.961 \\
\hline 500 & 0.755 & 0.805 & 0.963 \\
\hline
\end{tabular}

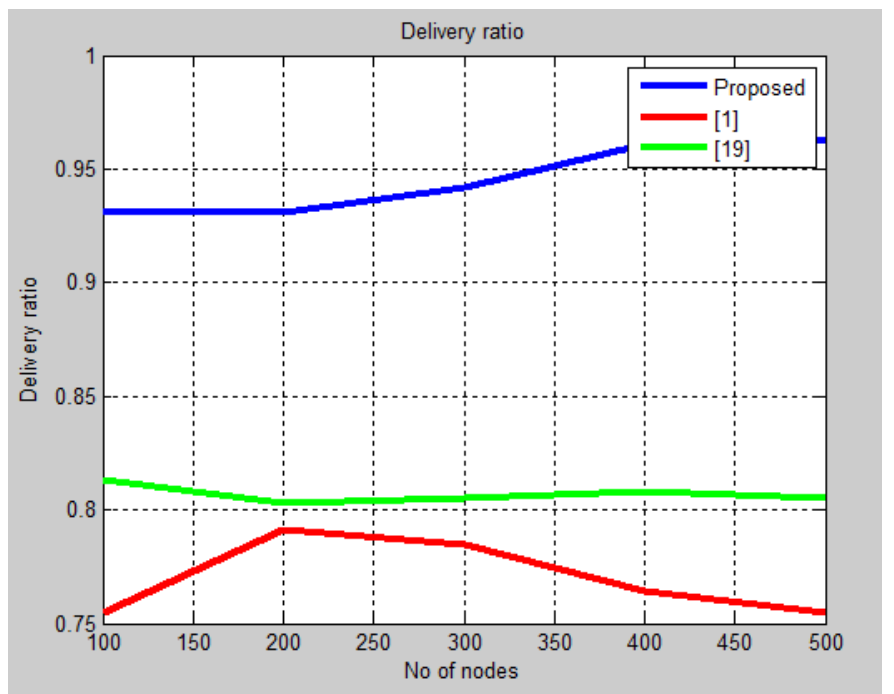




\section{Energy Efficient QoS guaranteed Cross Layer Solution (EEQCL) for Mesh backbone IOT networks}

The packet delivery ratio is higher in the proposed solution due to use of delay controlled route and data rate control in the selected route.

The lifetime of node is measured in terms of first node death from the start time of the simulation and the result is below

\begin{tabular}{|l|l|l|l|}
\hline Nodes & {$[1]$} & {$[19]$} & Proposed \\
\hline 100 & 4.1 & 4.7 & 6.4 \\
\hline 200 & 4.3 & 5.1 & 6.7 \\
\hline 300 & 4.9 & 5.3 & 7.1 \\
\hline 400 & 5.1 & 5.7 & 7.6 \\
\hline 500 & 5.3 & 6.0 & 8.1 \\
\hline
\end{tabular}

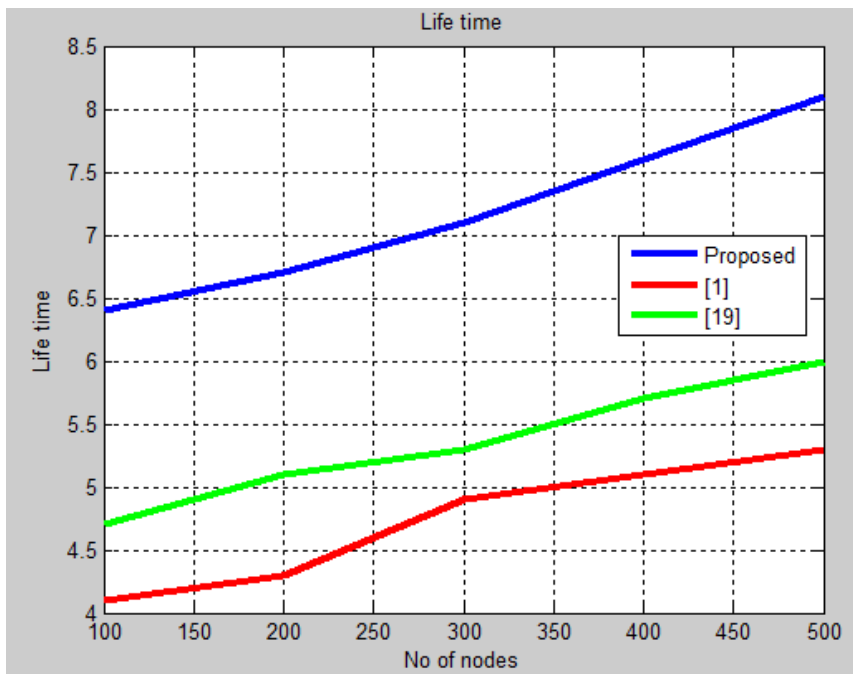

The life time is higher in the proposed solution due to controlled reactive routing within cluster, optimized routing across clusters, data rate and transmission rate control.

The contribution to life time due to transmission control is measured and given below

\begin{tabular}{|l|l|l|}
\hline Nodes & With control & $\begin{array}{l}\text { Without } \\
\text { control }\end{array}$ \\
\hline 100 & 6.4 & 5.8 \\
\hline 200 & 6.7 & 5.9 \\
\hline 300 & 7.1 & 6.1 \\
\hline 400 & 7.6 & 6.3 \\
\hline 500 & 8.1 & 6.5 \\
\hline
\end{tabular}

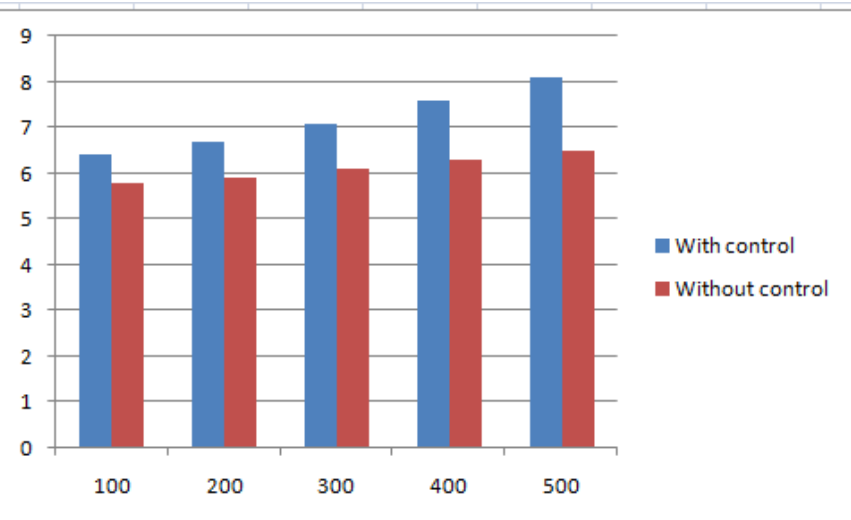

The contribution to life time due to data rate control is measured and given below

\begin{tabular}{|l|l|l|}
\hline Nodes & With control & $\begin{array}{l}\text { Without } \\
\text { control }\end{array}$ \\
\hline 100 & 6.4 & 6.1 \\
\hline 200 & 6.7 & 6.2 \\
\hline 300 & 7.1 & 6.4 \\
\hline 400 & 7.6 & 6.7 \\
\hline 500 & 8.1 & 7.0 \\
\hline
\end{tabular}

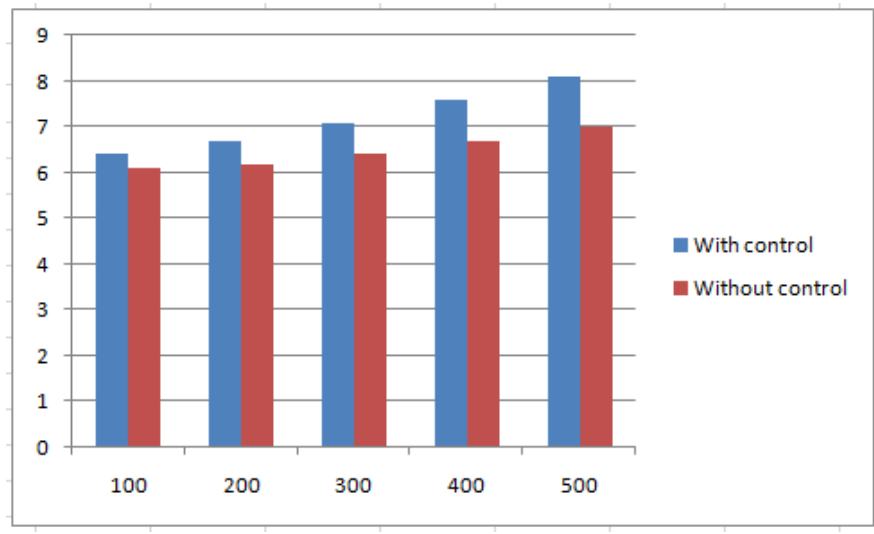

The life time has improved a lot due to data rate control and power control.

\section{CONCLUSION}

A cross layer based energy efficient QOS guaranteed solution is proposed for WMN based IOT networks. The solution involves topology controlled energy efficient composite routing at network layer, data control at application layer based on network feedback, differentiated service flow processing at session layer and multi channel interface with transmission control at the physical layer. The life time of proposed solution is higher than other recent cross layer solutions. The work also addressed data rate control for both text and image data.

\section{REFERENCES}

[1] Yang X, Wang L, Xie J, Zhang Z (2018) Cross-layer model design in wireless ad hoc networks for the Internet of Things. PLoS ONE 13(5) e0196818.

[2] Machado K, Rosário D, Cerqueira E "Routing Protocol Based on Energy and Link Quality for Internet of Things Applications",Sensors (Basel) 2013 Feb 4:13(2):1942-64

[3] K. Kumar,S. Kumar,"Distance, Energy and Link Quality Based Routing Protocol for Internet of Things",Conference on Signal, Networks, Computing, and Systems,Springer India 2017.

[4] Park, S., Cho,S., Lee, J.: "Energy-Efficient Probabilistic Routing Algorithm for Internet of Things," Journal of Applied Mathematics(213106)

[5] M. Yoon, "An energy-efficient routing protocol using message success rate in wireless sensor networks," KITCS/FTRA Journal of Convergence, vol. 4, no. 1, 2013

[6] M. M. A. Azim, "MAP: a balanced energy consumption routing protocol for wireless sensor networks," International Journal of Information Processing Systems, vol. 6, no. 3, 2010.

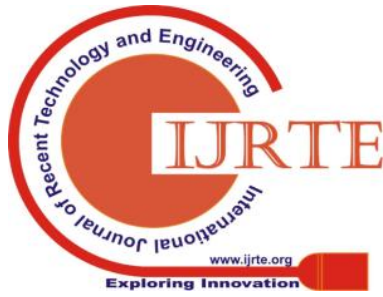


[7] M. Zhao, I. Ho, and P. H. J. Chong, "An Energy-efficient Region-based RPL Routing Protocol for Low-Power and Lossy Networks," IEEE Internet of Things Journal, pp. 1-1, 2016.

[8] B. F. Marques, M. P. Ricardo, "Improving the energy efficiency of WSN by using application-layer topologies to constrain RPL-defined routing trees", 2014 13th Annual Mediterranean Ad Hoc Networking Workshop (MED-HOC-NET), 2014

[9] S. A. Alvi, G. A. Shah, and W. Mahmood, "Energy efficient green routing protocol for Internet of Multimedia Things," 2015 IEEE Tenth International Conference on Intelligent Sensors, Sensor Networks and Information Processing (ISSNIP), 2015.

[10] M. Zhao, I. Ho, and P. H. J. Chong, "An Energy-efficient Region-based RPL Routing Protocol for Low-Power and Lossy Networks," IEEE Internet of Things Journal, pp. 1-1, 2016.

[11] K. Sindhuben, B. Rajiv, R. Tewari,"Cross Layer Energy Efficient Cost Link Based Routing for Wireless Sensor Network",Information and Communication Technologies (WICT), pp.804-809, 2011.

[12] Y. Peng, Y. Yu, L. Guo, D. Jiang, and Q. Gai, ”An efficient joint channel assignment and qos routing protocol for ieee 802.11 multi-radio multichannel wireless mesh networks," Elsevier Journal of Network and Computer Applications, 2012.

[13] Chen, Z., Wang, H., Liu, Y., Bu, F. and Wei, Z. (2012) A Context-Aware Routing Protocol on Internet of Things Based on Sea Computing Model. Journal of Computers, 7, 96-105.

[14] Rani, S., Talwar, R., Malhotra, J., Ahmed, S. H., Sarkar, M., \& Song, H A Novel Scheme for an Energy Efficient Internet of Things Based on Wireless Sensor Networks.Sensors(MDPI),2015,vol. a5(11), pp. 2860328626.

[15] Tang, J.; Zhou, Z.; Niu, J.; Wang, Q. An energy efficient hierarchical clustering index tree for facilitating time-correlated region queries in the internet of things. J. Netw. Comput. Appl. 2014, 40, 1-11..

[16] Long, J., Dong, M., Ota, K., Liu, A., \& Hai, S. Reliability guaranteed efficient data gathering in wireless sensor networks. IEEE Access,2015, vol. 3, pp. 430-444.

[17] M. Cheng, Q. Ye, and L. Cai, "Cross-layer schemes for reducing delay in multihop wireless networks," IEEE Transactions on Wireless Communications, vol. 12, no. 2, pp. 928-937, Feb. 2013.

[18] N. Ababneh, N. Timmons, and J. Morrison, "Cross-layer optimization protocol for guaranteed data streaming over wireless body area networks," in the Proc of the 8th International Wireless Communications and Mobile Computing Conference (IWCMC), 2012, pp. 118-123

[19] L. Shu, M. Hauswirth, Y. Zhang, J. Ma, G. Min, and Y. Wang, "Cross layer optimization for data gathering in wireless multimedia sensor networks within expected network lifetime," Journal of Universal Computer Science, vol. 16, no. 10, pp. 1343-1367, May 2010.

[20] M. O. Farooq, M. St-Hilaire, and T. Kunz, "Cross-layer architecture for qos provisioning in wireless multimedia sensor networks." TIIS, vol. 6 , no. 1 , pp. $178-202,2012$ 